\title{
On-Line Fault Detection in Wind Turbine Transmission System using Adaptive Filter and Robust Statistical Features
}

\author{
Ruoyu $\mathrm{Li}^{1}$ and Mark Frogley ${ }^{2}$ \\ ${ }^{1,2}$ SKF USA Remote Diagnostics Center,SKF USA Inc., Houston, TX 77086 \\ ruoyu.li@skf.com \\ mark.frogley@skf.com
}

\begin{abstract}
To reduce the maintenance cost, avoid catastrophic failure, and improve the wind transmission system reliability, online condition monitoring system is important. Developing effective online fault detection methodology is important. In this paper, an adaptive filtering technique is applied for enhancing the fault impulse signals-to-noise ratio in wind turbine gear transmission systems. Multiple statistical features designed to quantify the impulse signals of the processed signal are extracted for rotating machine fault detection. The multiple dimensional features are then transformed into one dimensional feature. A minimum error rate classifier will be designed based on the transformed one dimensional feature to identify the gear transmission system with defect. Vibration signals collected from wind turbines in the real operation will be used to demonstrate the effectiveness of the presented methodology.
\end{abstract}

Keywords: Adaptive filtering, Fault detection, Fault diagnosis, Condition monitoring, Gear transmission system, Statistical features, Pattern classification, Wind turbine transmission system.

\section{INTRODUCTION}

Wind Power is the world's fastest growing renewable energy source. With the developing and growing of wind power, reducing the cost of generating the wind energy becomes a critical issue. As wind turbines are often located in remote locations, the operation and maintenance costs are usually high. According to a survey on the failures of the Swedish wind turbines (Ribrant et al., 2007), more than $30 \%$ of the failures are mechanical failures. The repair costs of the mechanical failure are relatively high comparing to other failures such as sensor issue, and electric related issues (Hyers et al., 2006) (Nilsson and Bertling, 2007).

Ruoyu Li et al. This is an open-access article distributed under the terms of the Creative Commons Attribution 3.0 United States License, which permits unrestricted use, distribution, and reproduction in any medium, provided the original author and source are credited.
Thus deploying condition based monitoring (CBM) system could prevent catastrophic machine failure, improve the reliability and decrease the maintenance costs.

For wind turbine transmission system condition monitoring, different types of signals such as vibration, acoustic emission, temperature, oil debris, power performance and so on could be used (Han \& Song, 2005), (Zhu et al., 2012), (Schlechtingen et al., 2013), (Abouhnik \& Albarbar, 2012) Among them, vibration signals are currently widely used technique for mechanical fault detection and diagnosis. In the real applications, one type of rotating mechanical fault, for instance bearing surface defect, gear tooth crack, chipped gear tooth, generate impulse signals (McInerny and Dai, 2003) (Wang, 2001) (Endo, H., and Randall, 2007). When these faults develop inside rotating machinery, each time the rotating components pass over the damage point, an impact force will be created. The impact force will cause a ringing of the support structure at the structural natural frequency. By effectively detecting those periodic impulse signals, one group of rotating machine faults could be detected. In real complex machines it is not always possible to place the sensors directly on the rotating components. Thus the fault impulse signals collected by the sensors installed at some distance away from the rotating components are usually relatively weak and buried in the background noise and other rotating components, such as shaft, blades, and gears and so on. Moreover, wind turbine transmission systems work under dynamic operating conditions. The changing of rotating speed introduces smearing effects to the Fourier spectrum (Wang and Heyns, 2011). This will further increase the difficulties in fault detection and diagnostics. Finding a way to increase the impulse signal-to-noise ratio (SNR) is important for wind turbine transmission system fault detection. Another difficulty in online monitoring of wind turbine system is how to analyze the data in a large volume efficiently. For example, a typical condition monitoring system for wind turbine mechanical transmission consists about six to eight sensors on different locations and for each sensor, there are about two to four measurement readings with different setups. Usually every 10 to 120 minutes, different key 
features values will be collected and sent back to the server for trend analysis purpose and every 8-12 hours, time waveforms and spectrums will be captured and send back to the server for detail analysis purpose. For a wind farm with size of 100 turbines, when all the operating conditions met the requirement of activating the condition monitoring system, there will be about 7200 to 23040 extracted trend values for just one key feature and about 2400 to 9600 time waveforms usually with 4096 or 8192 sampling points and spectrums with 1600 to 6400 frequency lines daily for analyzing. Let us assume that it will take approximately 20 seconds to 30 seconds for an experienced human expert to analyze the spectrum for machine fault detection purpose. That means it will take a human vibration expert about 13 hours to 80 hours to analyze all the daily collected signals. Thus develop an effective automatic way on analyzing the signals and making decision will be in great need.

In this paper, an online automatic fault detection methodology has been developed. The methodology uses the adaptive filtering technique for enhancing the fault impulse signals-to-noise ratio in wind turbine gear transmission systems. Then multiple statistical features designed to quantify the impulse signals of the processed signal are extracted for detecting one type of rotating machine faults which generate impulse signals. The multiple dimensional features are transformed into one dimensional feature. A minimum error rate classifier is then designed to identify the type of faults which generate periodic impulses signals. Wind turbine vibration signals collected from the real operation will be used to demonstrate the effectiveness of the presented methodology. The remainder of the paper is organized as follows. Section 2 explains theoretical basis of the methodology. The analysis results on vibration signal collected from wind turbine systems are provided in Section 3. Section 4 summarizes the work done and concludes the paper.

\section{THEORETIC BASIS}

\subsection{Self-adaptive Noise Cancellation}

Self-adaptive noise cancellation (ANC) (Widrow et al., 2005) is used in this paper. Its structure is shown in Figure 1. The technique has been widely used in biological signal processing (Rahman et al., 2011), (Inan et al., 2010), (Thakor \& Zhu, 1991) to remove the electrical interference, in the audio signal processing (Greenberg, 1998), (Sambur, 1978) to improve the quality of interested voice signal, in the non-destructive testing area (Zhu \& Weight, 1994) to improve the SNR for the acoustic emission signal, and the vibration signal based rotating machine fault detection (Li \& He, 2011), (Antoni \& Randall, 2004), and (Bechhoefer et al., 2009) to enhance the impulse fault SNR.

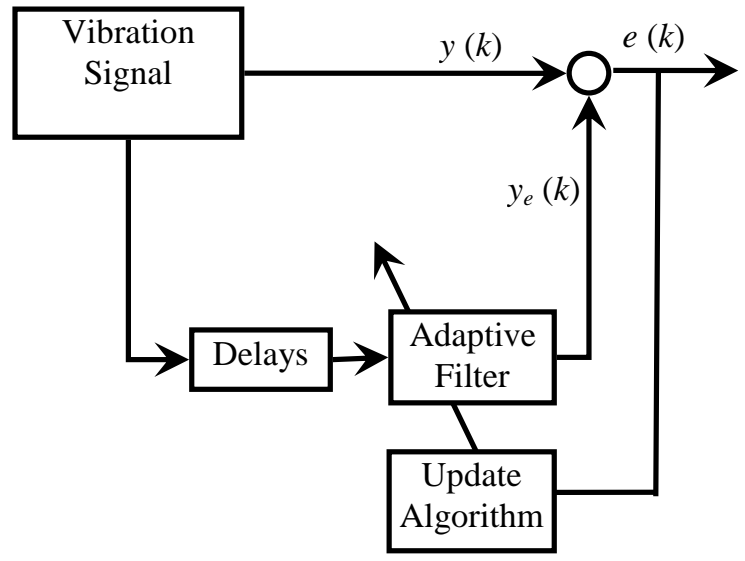

Figure 1. The scheme of the adaptive noise cancellation

The idea here is to use the adaptive filter to track the periodic components inside the signal and then remove it from the original vibration signal. The parameters of the filter are adaptively updated by using the error signal, expressed in Eq. (1). The way for calculating the optimal parameters of the prediction model is to minimize the mean squared error between the original signal and the predicted signal.

$$
e_{k}=y(k)-y_{e}(k)
$$

where $y(k)$ is the input vibration signal at time $k, y_{e}(k)$ is the estimated output signal, $e_{k}$ is error between the estimated signal and the input signal at time $k$.

To adaptively adjust the coefficient of the prediction model, the least mean squared algorithm is applied to update the coefficients online. The update equations, presented in section 1 of the book (Benesty \& Huang, 2003) are shown in Eq. (2) and Eq. (3).

$$
\begin{aligned}
& y_{e}(n)=W_{k}^{T} X_{k} \\
& W_{k+1}=W_{k}+2 \mu e_{k} X_{k}
\end{aligned}
$$

where, $W_{k}=\left[w_{1}(k), w_{2}(k), \ldots, w_{l}(k)\right]$ is the parameters vector of the adaptive filter at time $k, l$ is the length of the filter, $X_{k}=[y(k-\Delta), y(k-\Delta-1), \ldots y(k-\Delta-(l-1))] \quad$ is the delayed version of input $\mathrm{y}(\mathrm{k}), \Delta$ is the delay time and is $\mu$ is the learning step size.

A simulation is used here for demonstration purpose. A sine waveform, impulses and Gaussian noise are used to compose the simulated signal. The adaptive noise cancellation was then applied to the simulated signal. Both the simulated signal and the processed signal are shown in Figure 2. In Figure 2, x-axis represents the time and y-axis represents the amplitude of the signal. The top red curve stands for the simulated signal. The blue curve stands for the processed signal. From Figure 2, one could easily see 
that the adaptive noise cancellation algorithm effectively remove the periodic component, the sine waveform.

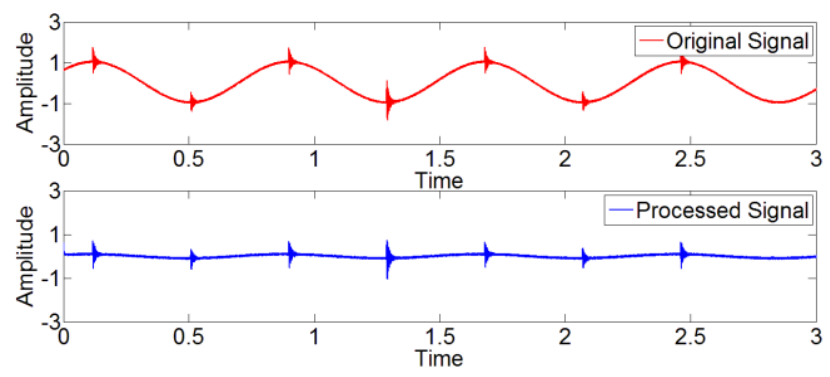

Figure 2. The simulated signal and the processed signal

\subsection{Statistical Feature extraction}

To design a classifier, quantification values are needed. As the purpose in this paper is for impulse signal detection, five statistical values commonly used for rotating machine fault detection and diagnosis (Lei et al., 2008) (Samanta, et al., 2001) (Medjaher et al., 2012), were used here to quantify the processed impulse signal enhanced signal. They are Kurtosis, Crest Factor, Root Mean Square (RMS), Impulse Factor, and Skewness (Norton et al., 2003).

Kurtosis is the fourth statistical moment. It is a good indication of the "peakedness" of the signal. The Kurtosis is defined as,

$$
x_{K T}=\frac{\frac{1}{n} \sum_{i=1}^{n}\left(x_{i}-\bar{x}\right)^{4}}{\left(\frac{1}{n} \sum_{i=1}^{n}\left(x_{i}-\bar{x}\right)^{2}\right)^{2}}
$$

The Crest Factor is the ratio of the peak value to the RMS value. It is a good measurement of spikiness of a signal. The Crest Factor is defined as,

$$
x_{C R}=\frac{\max \left|x_{i}\right|}{\sqrt{\frac{1}{n} \sum_{i=1}^{n} x_{i}^{2}}}
$$

The RMS value is usually used to represent the overall energy of the signal. The RMS is defined as,

$$
x_{r m s}=\sqrt{\frac{1}{n} \sum_{i=1}^{n} x_{i}^{2}}
$$

The Impulse factor is the ratio of the absolute peak value to the absolute mean value of the signal. It is sensitive for detection of bearing problem (Yiakopoulos et al., 2011). The Impulse factor is defined as,

$$
x_{I F}=\frac{\max \left|x_{i}\right|}{\frac{1}{n} \sum_{i=1}^{n}\left|x_{i}\right|}
$$

The Skewness is a third statistical moment. Skewness is usually used to measure the symmetry of the signal. The Skewness is defined as,

$$
x_{K T}=\frac{\frac{1}{n} \sum_{i=1}^{n}\left(x_{i}-\bar{x}\right)^{3}}{\left(\sqrt{\frac{1}{n} \sum_{i=1}^{n}\left(x_{i}-\bar{x}\right)^{2}}\right)^{3}}
$$

In Eq. (4) to (8), $x$ is the signal with $n$ samples. $\bar{x}$ is the mean value of $x$.

\subsection{Linear Discriminant Analysis}

Linear discriminant analysis (LDA) (McLachlan, 2004) is a well known technology which is widely used for feature extraction and dimension reduction. Many successful applications are presented in various research papers (Swets and Weng, 1996) (Martinez and Kak, 2001) (Lu et al., 2003). LDA projects the data sets with multiple classes into a lower-dimensional vector, which provides maximized separation between the classes. In this paper, as only two classes, health state and fault state are considered, multiple class LDA will not be discussed and only two classes LDA will be considered.

Let's assume that we have a set of $d$-dimensional $n$ samples $s=\left[s_{1}, s_{2}, \ldots, s_{n}\right] . N_{1}$ of them belong to class $C_{1} . N_{2}$ of them belong to class $C_{2}$. If we define a transformation matrix $w$, then we could obtain a scalar $y$ by projecting the $s$ onto a line,

$$
y=w^{T} s
$$

$y$ is a set of $n$ samples $y=\left[y_{1}, y_{2}, \ldots, y_{n}\right] . N_{1}$ of them belong to class $C_{T 1} . N_{2}$ of them belong to class $C_{T 2}$.

To find the scalar which maximizes the separation between the two classes, the following objective function is adopted (McLachlan, 2004). By maximizing the objective function in Eq. (9), we could obtain the projecting which provides best separation between the two classes.

$$
J(w)=\frac{\left|\tilde{\mu}_{1}-\tilde{\mu}_{2}\right|^{2}}{\tilde{s}_{1}^{2}+\tilde{s}_{2}^{2}}
$$

Where

$$
\begin{aligned}
& \tilde{\mu}_{i}=\frac{1}{N_{i}} \sum_{s \in C_{T_{i}}} w^{T} s=w^{T} \mu_{i} \text { and } \\
& \tilde{s}_{i}^{2}=\sum_{y \in C_{T_{i}}}\left(y-\tilde{\mu}_{i}\right)^{2}, i=[1,2] .
\end{aligned}
$$

Through maximizing process, the transform matrix could be calculated as, 


$$
w=S_{W}^{-1}\left(\mu_{1}-\mu_{2}\right)
$$

Where

$$
\begin{aligned}
& \mu_{i}=\frac{1}{N_{i}} \sum_{s \in C_{i}} w^{T} s, i=[1,2] \text { and } \\
& S_{W}=\sum_{i=1}^{2} \sum_{s \in C_{i}}\left(s-\mu_{i}\right)\left(s-\mu_{i}\right)^{T}
\end{aligned}
$$

\section{DAta Collection AND EXPERIMENTAL RESUltS}

Recently, a number of SKF IMx-W, WinCon units (SKF, 2011) were installed on a fleet of wind turbines to perform the online condition monitoring. The SKF IMx-W, WinCon unit is a modern industrial product specialized designed for on-line vibration monitoring. It has been successfully applied to online monitor the wind turbine mechanical faults all around the world. The key features of the system include (SKF, 2011):

(1) Lightning protection.

(2) Wall-mounted.

(3) Sixteen analogue inputs and two digital inputs.

(4) Simultaneous measurement of all channels.

(5) Multi-parameter gating.

The monitored wind turbine mechanical transmission systems consist of main bearing, gear transmission system and generator. A simplified transmission system with one planetary gear stage is shown in Figure 3. The monitoring system used two accelerometers on the main bearing, four on the gearbox, and two on the generator. The system also measured the rotating speed of the high speed shaft to provide the speed reference.

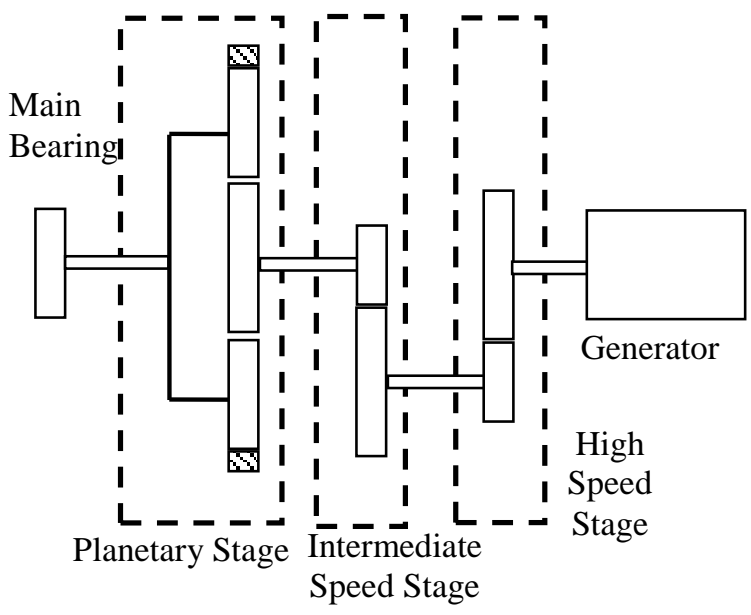

Figure 3. The simplified typical wind turbine transmission system
During our daily monitoring process, bearing defects on the intermediate shaft from four wind turbines have been captured. After the detection, the bearings have been scheduled to replace with the new bearing of the same type. In this paper, the acceleration signal collected from the intermediate shaft of four of the wind turbines with bearing issue will be investigated. The SKF series accelerometers were used for the data collection. This type of sensor has $100 \mathrm{mv} / \mathrm{g}$ sensitivity and the frequency range is about $0.5 \mathrm{~Hz}$ to $10 \mathrm{kHz}$. The sampling frequency for the intermediate acceleration measurements were set to be $5120 \mathrm{~Hz}$ and about 1.6 seconds long signal was collected for each data collection. Routinely, the vibration signals will be collected every 8 hours during the normal daily collection.

For analysis purpose, 20 vibration signals before the replacement and after the replacement were randomly selected from the database of each turbine from the signals collected during the past 6 month. A typical vibration signal collected from a healthy gearbox is shown in Figure 4 and that collected from a gearbox with defect is shown in Figure 5. Figure 6 shows the picture of the damaged bearing inner race. The crack on the surface could be clearly seen from both figures.

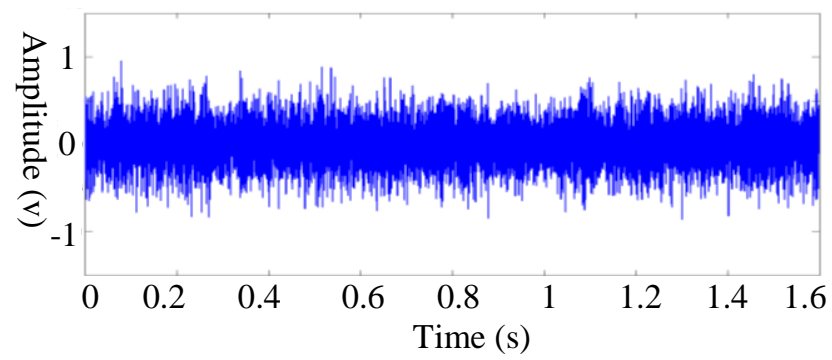

Figure 4. The vibration signal collected from a healthy gearbox

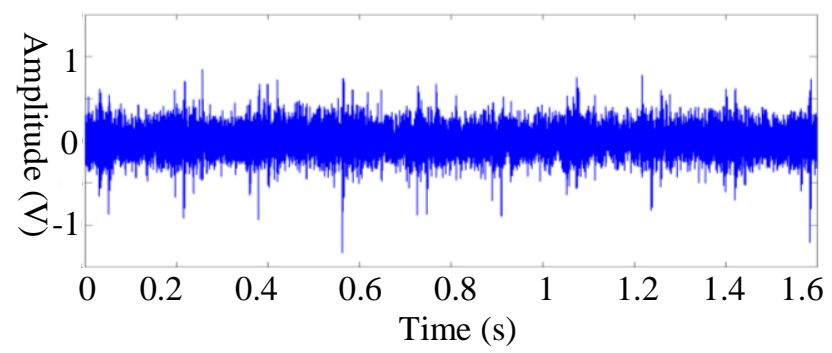

Figure 5. The vibration signal collected from a gearbox with bearing inner race defect

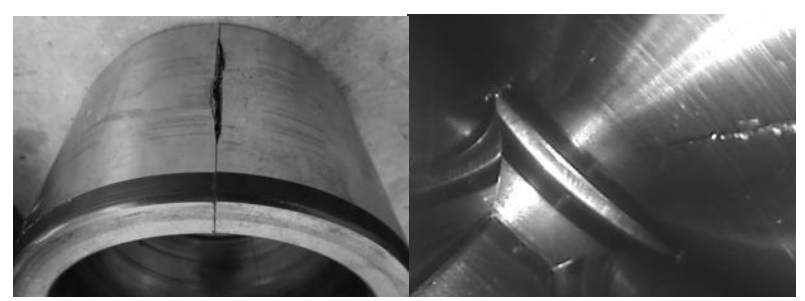

(a)

(b) 


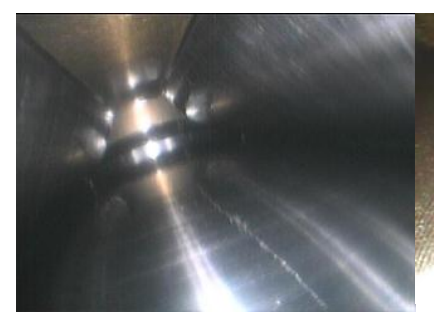

(c)

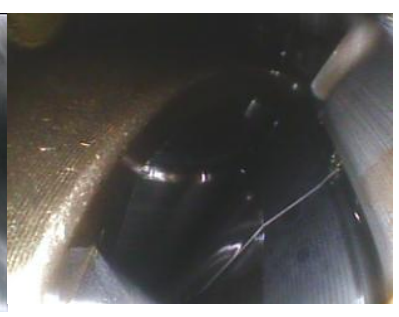

(d)
Figure 6. (a) Damaged Bearing with Crack on the Whole Inner Race of Wind Turbine \#1. (b) Damaged Bearing with Crack on the Surface the Inner Race of Wind Turbine \#2.

(c) Damaged with hair line Crack on the Surface of the Inner Race of Wind Turbine \#3. (d) Damaged with Crack on the Surface of the Inner Race of Wind Turbine \#4

The ANC was applied to process the vibration signals and sample results of the vibration from the healthy state and the damaged state are shown in Figure 7 and Figure 8.

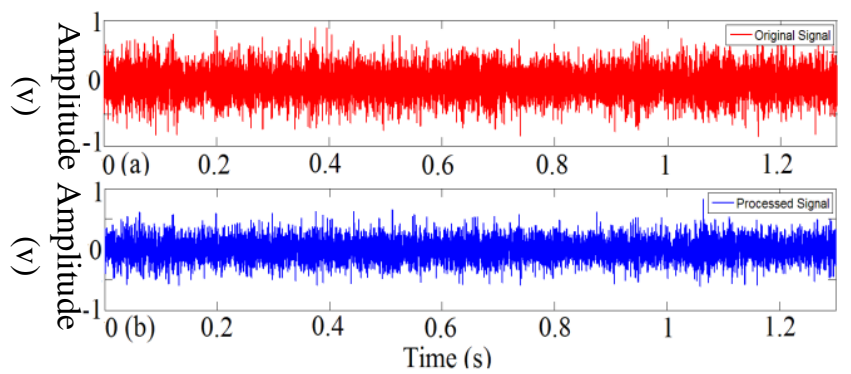

Figure 7. The processed results of the healthy gearbox

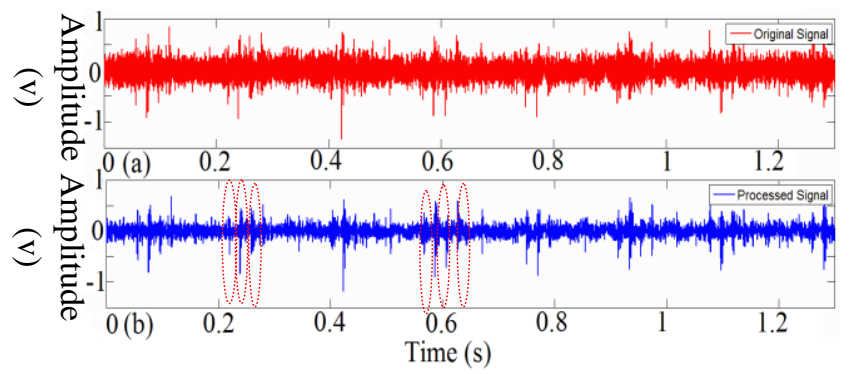

Figure 8. The processed results of the gearbox with bearing inner race defect

Comparing the results shown in Figure 7 (b) and Figure 8 (b), one could easily see the periodic fault impulses in Figure 8 (b). The periodic frequency of those impulses is related to the bearing inner race defect. This is a clear indication of there being a bearing inner race defect. In Figure 8 (a), one could see some of the impulse signals appearing in the original vibration signal, but due to the low impulse SNR, the analysis on the time waveform is hard to find the periodic behavior of the impulses. However, from the processed results shown in Figure 8 (b), the impulses signals (the red circled one) could be easily identified. By simple analysis on processed time wave form, the potential fault could be identified.
The comparison between the ANC and the widely used envelope analysis (McInerny and Dai, 2003) are investigated in this paper. The bandwidth of the band-pass filter of the envelope analysis is important and many research papers have presented ways to select the optimal bandwidth (Sawalhi et al., 2007) (Eric et al., 2011). However, this is beyond the research scope of this paper. In this paper, the bandwidth is set to between $500 \mathrm{~Hz}$ and 2 $\mathrm{kHz}$. Ten vibration signals collected on the wind turbines with known bearing fault have been randomly selected. For demonstration purpose, the Fourier spectrums of both algorithms of one of the bearing fault signals were shown in Figure 9 (a) and (b). Figure 9 (a) shows the Fourier spectrum of the envelope analysis and Figure 9 (b) shows the Fourier spectrum of the ANC. From Figure 9 (a) and (b), the high peak at the bearing inner race defect frequency and its harmonics could be easily observed on both the spectrum. Both algorithms effectively capture the bearing defect.
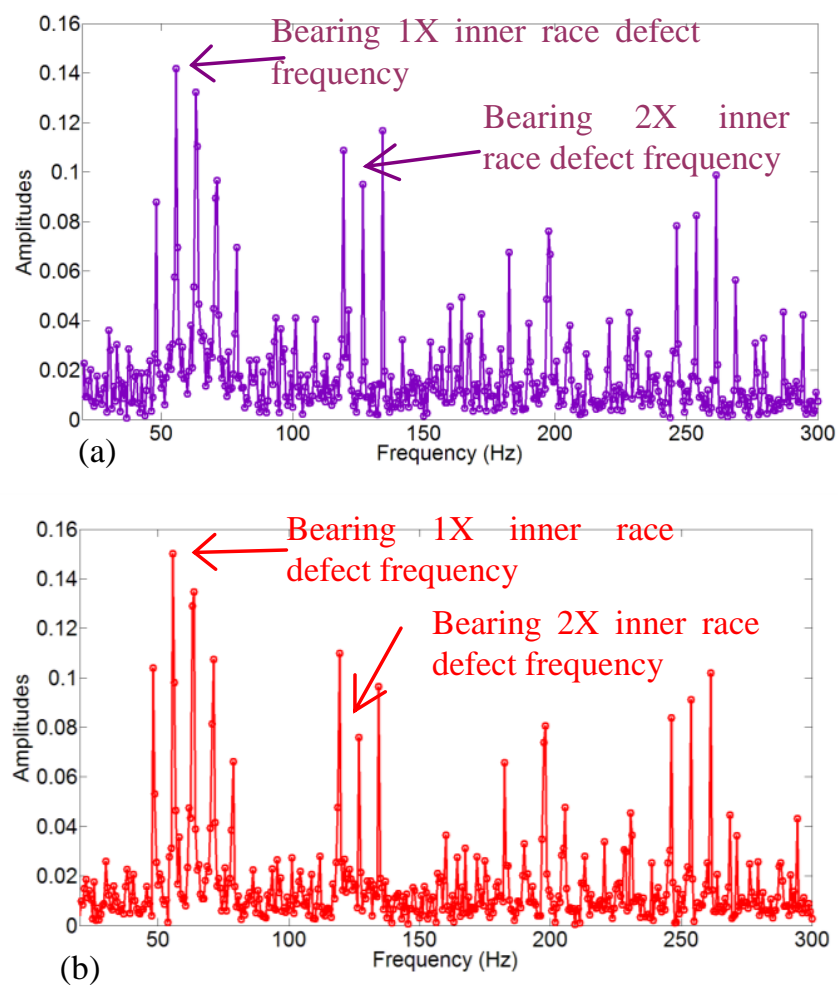

Figure 9. The spectrum of (a) the envelope analysis and (b) the adaptive noise cancellation algorithm

To quantify the performance of the two algorithms, the quantification value is calculated by the following equation.

$$
I_{q}=\frac{\sum_{i=1}^{2} A_{i}}{S_{R M S}}
$$


where $A_{i}$ is the amplitude of the $i^{\text {th }}$ harmonics of the bearing fault frequency of the spectrum and $S_{R M S}$ is the RMS value of the spectrum.

In our case, as the bearing is with inner race defect, the inner race defect frequency of the bearing is used for calculation. The higher the $I_{q}$ value is the better performance the algorithm has. The results of the $I_{q}$ value are shown in Table 1. The percentage of the difference between $I_{q}$ values is the ratio of the subtraction of $I_{q}$ of the adaptive noise cancellation from that of the envelope analysis to the $I_{q}$ of the envelope analysis. From the results shown in Table 1, one could see that adaptive noise cancellation algorithm has slightly higher $I_{q}$ value of the traditional envelope analysis.

Table 1. The $I_{q}$ value of the traditional envelope analysis and the adaptive noise cancellation

\begin{tabular}{|c|c|c|c|c|c|c|}
\hline \multicolumn{2}{|c|}{ Healthy Gearbox } & \multicolumn{3}{c|}{ Gearbox with Defect } & $\begin{array}{c}\text { Normalized } \\
\text { Difference }\end{array}$ \\
\hline & Mean & STD & & Mean & STD & \\
\hline Kurtosis & 2.9413 & 0.2080 & Kurtosis & 6.7379 & 1.8137 & 1.2908 \\
\hline $\begin{array}{c}\text { Crest } \\
\text { Factor }\end{array}$ & 8.3849 & 1.7355 & Crest Factor & 12.8863 & 1.6221 & 0.5368 \\
\hline RMS & 2.0668 & 1.0749 & RMS & 1.6640 & 0.6494 & 0.1949 \\
\hline $\begin{array}{c}\text { Impulse } \\
\text { Factor }\end{array}$ & 10.5101 & 2.1233 & $\begin{array}{c}\text { Impulse } \\
\text { Factor }\end{array}$ & 17.6538 & 2.7323 & 0.6797 \\
\hline Skewness & -0.0181 & 0.0892 & Skewness & -0.0545 & 0.1636 & 2.0110 \\
\hline
\end{tabular}

The Kurtosis, Crest Factor, Impulse Factor, RMS, and Skewness of the processed signal were calculated for both the healthy vibration signal and the vibration signal with damaged components. The mean and the standard deviation value of the statistical values were shown in Table 2 . Those feature values were then transformed into one dimensional feature vector by using the LDA. To quantify how well the individual statistical features, listed in Table 2, separate the healthy states from the fault states, the percentage of the difference between the two states are calculated. The percentage of the difference is the ratio of the absolute difference between the healthy states and the fault states to the feature value of the healthy states. The values are shown in Table 2. To obtain a general ideal on the computational requirement of the developed methodology, tests were conducted on a pc with a $2.60 \mathrm{GHz} \mathrm{CPU}$ and 3 GB RAM. By processing the 8192 point vibration signals, the processing time was approximately 0.5 seconds.

The histogram of the transformed feature of both the healthy machine and the damaged machine is shown in Figure 10.
In Figure 10, the $\mathrm{x}$-axis represents the amplitude of the transformed feature. The $y$-axis represents the numbers of the machine in the group. The purple group stands for the fault states and the red group stands for the healthy states.

Table 2. The calculated values of the processed signal of healthy gearbox and the gearbox with defect

\begin{tabular}{|c|c|c|}
\hline $\begin{array}{c}\text { Traditional } \\
\text { Envelope }\end{array}$ & $\begin{array}{c}\text { Adaptive } \\
\text { Noise } \\
\text { Cancellation }\end{array}$ & $\begin{array}{c}\text { Percentage of } \\
\text { difference (\%) }\end{array}$ \\
\hline 16.33 & 16.36 & 0.16 \\
\hline 15.50 & 16.30 & 5.18 \\
\hline 12.93 & 14.68 & 13.57 \\
\hline 11.92 & 12.91 & 8.29 \\
\hline 16.15 & 17.94 & 11.05 \\
\hline 11.54 & 13.21 & 14.48 \\
\hline 17.72 & 19.29 & 8.85 \\
\hline 17.27 & 18.00 & 4.22 \\
\hline 14.51 & 15.30 & 5.46 \\
\hline 13.50 & 14.27 & 5.71 \\
\hline
\end{tabular}

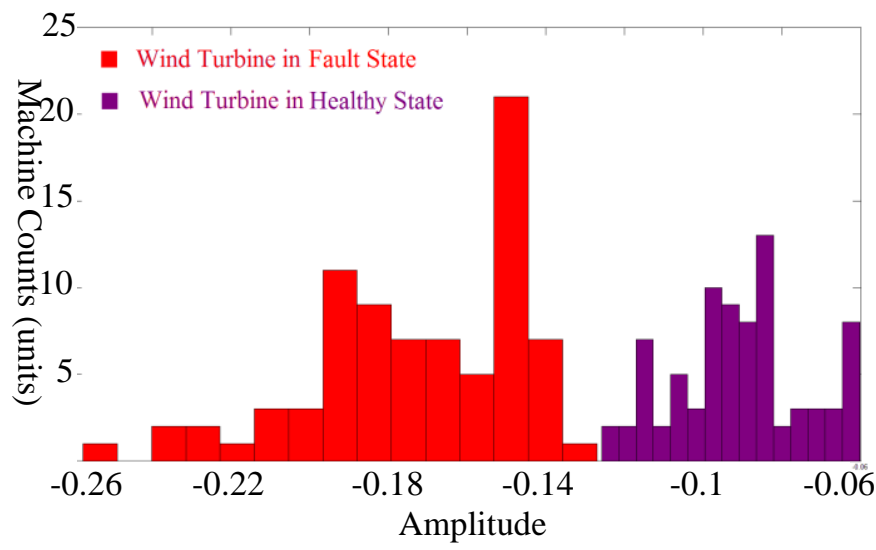

Figure 10. The histogram of the transformed feature of the healthy machine and the machine with damaged components

The decision boundary is then determined by the minimum error rate rule. As in this paper, only two classes, healthy state and damaged state are used, the prior probability determines how likely the unknown observation belongs to each state. For example, if we define the prior probability value to be 0.5 . Then the probability of the unknown extracted feature belongs to healthy state is 0.5 and that of the damage state is 0.5 . In this paper, three different boundaries were calculated by using three different prior probability values. They are $0.1,0.5$, and 0.9 . The results are shown in Figure 11. In Figure 11, the $\mathrm{x}$-axis represents the number of the samples. The y-axis represents the amplitude of the transformed feature. Four different shapes 
are used to represent different wind turbines. The stars, circles, diamonds, and squares represent wind turbine \#1, wind turbine \#2, wind turbine \#3, and wind turbine \#4, respectively. The color of the shapes represents the state of the wind turbine. The red represents the wind turbine is in healthy state. The purple represents the wind turbine in fault state. The blue dash line represents the decision boundary for 0.1 . The value of the boundary is -0.070 . The green dot line represents the decision boundary for 0.5 . The value of the boundary is -0.081 . The pink solid line represents the decision boundary for 0.9 . The value of the boundary is -0.088 . From Figure 11, one could see that the boundary for 0.1 is the most fault sensitive boundary among the three boundaries which identified several healthy states as damaged state. The boundary for 0.9 is the most fault insensitive boundary among the three boundaries which misclassified several damaged states as healthy state.

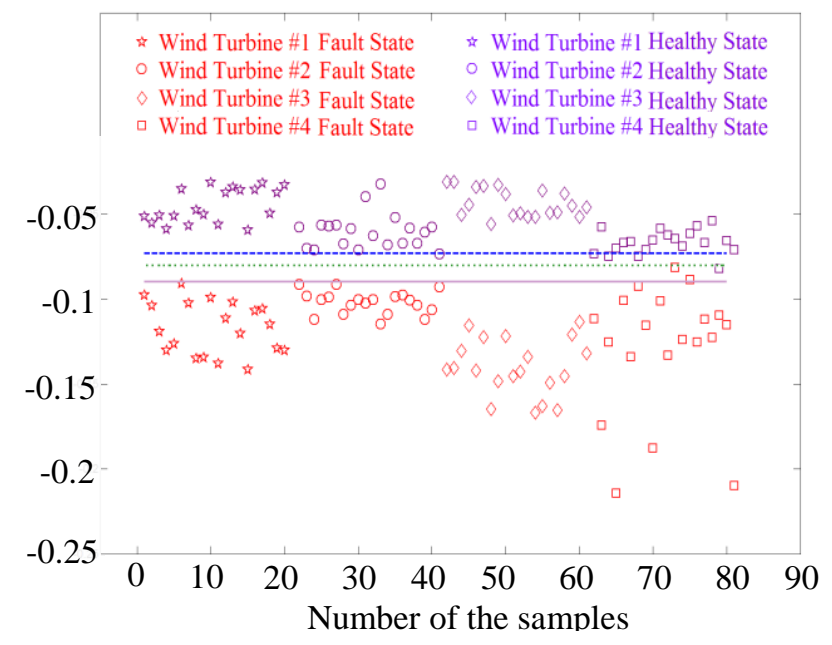

Figure 11. The transformed feature and the decision boundaries with different prior statistics (blue line: $\mathrm{P}($ Healthy $)=0.1$, green line: $\mathrm{P}($ Healthy $)=0.5$, pink line: $\mathrm{P}($ Healthy $)=0.9)$

The confusion matrix of three decision boundaries is shown in Table 3. In real online condition monitoring applications with large volume of data, determination of the decision boundary is important. Fault sensitive boundary will potentially cause more false alarms while fault insensitive boundary tends to miss the true faults. Thus determination of the boundaries in real applications is based on the following rule. When the system is deployed to a newly created wind farm, the less fault sensitive boundary should be used. With aging of the wind farm, the boundary will be adjusted to more fault sensitive one.
Table 3. The confusion matrix of the different boundaries

\begin{tabular}{|c|c|c|c|}
\hline & & $\begin{array}{c}\text { Calculated } \\
\text { Healthy }\end{array}$ & $\begin{array}{c}\text { Calculated } \\
\text { Fault }\end{array}$ \\
\hline $\begin{array}{c}\text { 0.1 } \\
\text { Boundary }\end{array}$ & True Healthy & 75 & 5 \\
\cline { 2 - 4 } & True Fault & 0 & 80 \\
\hline $\begin{array}{c}\mathbf{0 . 5} \\
\text { Boundary }\end{array}$ & True Healthy & 80 & 0 \\
\cline { 2 - 4 } & True Fault & 1 & 79 \\
\hline $\begin{array}{c}\mathbf{0 . 9} \\
\text { Boundary }\end{array}$ & True Healthy & 80 & 0 \\
\cline { 2 - 4 } & True Fault & 2 & 78 \\
\hline
\end{tabular}

From Table 3, one could see that $96.875 \%, 99.375 \%$, and $98.750 \%$ of accuracy are achieved for $0.1,0.5$, and 0.9 boundaries, respectively.

\section{Conclusions}

In real applications, effective fault detection algorithms are an essential part of the condition monitoring system, especially for the online continuous monitoring systems, like wind turbine condition monitoring systems. In this paper, an effective automatic fault detection methodology has been developed. The methodology uses adaptive filtering technique to improve the fault SNR and LDA to reduce the features' dimensions. Wind turbine vibration signals obtained in real operation were used to demonstrate the effectiveness of the presented methodology.

Currently, the developed methodology will continue testing on the vibration signals of wind turbines with different designs. In future work, it will be interesting to study the effectiveness of the developed method on a variety of rotating equipments in different industries and applications, such as ranging arms in the mining industries, pumps in the paper mill, and motors in the food industries, and so on.

\section{REFERENCES}

Abouhnik, A., and Albarbar, A., "Wind turbine blades condition assessment based on vibration measurements and the level of an empirically decomposed feature", Energy Conversion and Management, vol. 64, pp. 606613, 2012.

Antoni, J., and Randall, B., "Unsupervised noise cancellation for vibration signals: part I--evaluation of adaptive algorithms," Mechanical Systems and Signal Processing, vol. 18, pp. 89-101, 2004.

Bechhoefer, E., Li, R., and He, D., "Quantification of condition indicator performance on a split torque gearbox", Proceedings of the 2009 AHS Forum, Grapevine, TX, May 27-29, 2009.

Bechhoefer, E., Menon, P., and Kingsley, M., "Bearing envelope analysis window selection Using spectral kurtosis techniques", In 2011 IEEE Conference on 
Prognostics and Health Management (PHM), pp. 1-6, 2011.

Benesty, J., and Huang, Y., Adaptive signal processing: applications to real-world problems: Springer, 2003.

Greenberg, J. E., "Modified LMS algorithms for speech processing with an adaptive noise canceller," IEEE Transactions on Speech and Audio Processing, vol. 6, pp. 338-351, 1998.

Han, Y., and Song Y.H., "Condition monitoring techniques for electrical equipment-a literature survey", IEEE Transactions on Power Delivery, vol. 18, pp, 4-13, 2003

Inan, O. T., Etemadi, M., Widrow, B., and Kovacs, G., "Adaptive cancellation of floor vibrations in standing ballistocardiogram measurements using a seismic sensor as a noise reference," IEEE Transactions on Biomedical Engineering, vol. 57, pp. 722-727, 2010.

Lei, Y., He, Z., and Zi, Y. "A new approach to intelligent fault diagnosis of rotating machinery", Expert Systems with Applications, vol. 35, No. 4, 1593-1600, 2008.

Li, R., and He, D., "Development of an Advanced Narrowband Interference Cancellation Method for Gearbox Fault Detection," in AHS 67th Annual Forum and Technology Display, Virginia Beach, Virginia, 2011.

Lu, J., Plataniotis, K. N., and Venetsanopoulos, A. N., Face recognition using LDA-based algorithms., IEEE Transactions on Neural Networks, vol. 14, No.1, pp. 195-200, 2003.

Martinez A., and Kak A., "PCA versus LDA", IEEE Transactions on Pattern Analysis and Machine Intelligence, vol. 23, no. 2, pp. 228-233, 2001

McInerny, S. A., and Dai, Y., "Basic vibration signal processing for bearing fault detection", IEEE Transactions on Education, vol. 46, No. 1, 149-156, 2003.

McLachlan, G. J., Discriminant analysis and statistical pattern recognition, vol. 544, Wiley-Interscience, 2004

Medjaher K., Camci F., and Zerhouni N., "Feature Extraction and Evaluation for Health Assessment and Failure Prognostics", In First European Conference of the Prognostics and Health Management Society., pp. 111-116. 2012.

Norton, M. P., \& Karczub, D. G., Fundamentals of noise and vibration analysis for engineers. Cambridge university press. 2003

Rahman, M. Z. U., Rafi, A.S., and Rama, K.R., "Efficient sign based normalized adaptive filtering techniques for cancellation of artifacts in ECG signals: Application to wireless biotelemetry," Signal Processing, vol. 91, pp. 225-239, 2011.

Ribrant, J., and Bertling, L.M., "Survey of Failures in Wind Power Systems With Focus on Swedish Wind Power Plants During 1997-2005," Energy Conversion, IEEE Transactions on, vol.22, no.1, pp.167,173, 2007

Samanta, B., Al-Balushi, K. R., and Al-Araimi, S. A., "Artificial neural networks and support vector machines with genetic algorithm for bearing fault detection, Engineering Applications of Artificial Intelligence, vol. 16, No. 7, pp. 657-665, 2003

Sambur, M., "Adaptive noise canceling for speech signals," IEEE Transactions on Acoustics, Speech and Signal Processing, vol. 26, pp. 419-423, 1978.

Sawalhi, N., Randall, R. B., and Endo, H., "The enhancement of fault detection and diagnosis in rolling element bearings using minimum entropy deconvolution combined with spectral kurtosis", Mechanical Systems and Signal Processing, vol. 21, No. 6, pp. 2616-2633., 2007.

Schlechtingen M., Santos I.F., and Achiche S., "Wind turbine condition monitoring based on SCADA data using normal behavior models. Part 1: system description", Applied soft Computing, vol. 13, pp. 259270, 2013.

SKF, SKF Multilog On-line System IMx-W, www.SKF.com,2011

Swets, D. and Weng, J., "Using discriminant eigenfeatuers for image retrieval", IEEE Transactions on Pattern Analysis and Machine Intelligence, vol. 18, no. 8, pp. 831-836, 1996.

Thakor, N. V. and Zhu Y., "Applications of adaptive filtering to ECG analysis: noise cancellation and arrhythmia detection," IEEE Transactions on Biomedical Engineering, vol. 38, pp. 785-794, 1991.

Widrow, B., Glover, J., McCool, J., Kaunitz, J., Williams, C.S., Hearn, R.H., Zeidler, J.H., Dong, E., and Goodlin, R.C., "Adaptive noise cancelling: Principles and applications," Proceedings of the IEEE, vol. 63, pp. 1692-1716, 2005.

Yiakopoulos, C.T., Gryllias, K.C., and Antoniadis, I.A., "Rolling element bearing fault detection in industrial enviroments based on a k-mean clustering approach", Expert Systems with Applications, vol. 38, No. 3, pp. 2888-2911, 2011

Zhu J., He D., and Bechhoefer E., "Survey of lubrication oil condition monitoring, diagnostics, and prognostics techniques", Proceedings of the 2012 Conference of he Society for Machinery Failure Prevention Technology, pp. 193-212, Dayton, OH, April 24-26, 2012.

Zhu, Y., and Weight J.P., "Ultrasonic nondestructive evaluation of highly scattering materials using adaptive filtering and detection," IEEE Transactions on Ultrasonics, Ferroelectrics and Frequency Control, vol. 41, pp. 26-33, 1994.

Wang, W., "Early detection of gear tooth cracking using the resonance demodulation technique", Mechanical Systems and Signal Processing, vol. 15, No. 5, pp. 887903, 2001

Endo, H., and Randall, R. B., "Enhancement of autoregressive model based gear tooth fault detection technique by the use of minimum entropy deconvolution 
filter", Mechanical Systems and Signal Processing, vol. 21, No. 2, pp. 906-919, 2007

Wang, K., and Heyns, P. S., "The combined use of order tracking techniques for enhanced Fourier analysis of order components", Mechanical systems and signal processing, vol. 25, No. 3, pp. 803-811, 2011.

Hyers, R. W., McGowan, J. G., Sullivan, K. L., Manwell, J. F., and Syrett, B. C., "Condition monitoring and prognosis of utility scale wind turbines", Energy Materials: Materials Science and Engineering for Energy Systems, vol. 1, No. 3, pp. 187-203, 2006.

Nilsson, J., and Bertling, L., “ Maintenance management of wind power systems using condition monitoring systems - life cycle cost analysis for two case studies", IEEE Transactions on Energy Conversion, vol. 22, No. 1, pp. 223-229, 2007.

\section{BIOGRAPHIES}

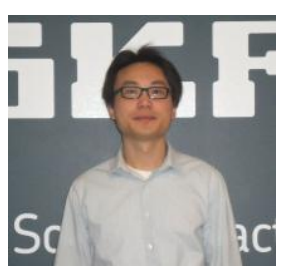

Ruoyu Li received the B.S. degree in automatic control and M.S. degree in control theory and control engineering from Guilin University of Electronic Technology, Guilin, China, in 2002 and 2005, respectively, and the Ph.D. at Chicago, Chicago, IL. degree from the University of Illinois

$\mathrm{He}$ is currently a reliability engineer with the Remote Diagnostic Center, SKF USA Inc., Houston, TX. His research interests include statistical signal processing, machine learning, data mining, rotating machinery fault diagnostics and prognostics, sensor networks, and mechatronics systems design.

Mark Frogley has worked in the field of rotating machinery condition monitoring and asset preventive maintenance since 1993. He has more than 15 years' experience on setting up and operating large predictive maintenance programs. Worked with SKF for more than 15 years, he is currently a technology integration manager with SKF USA Inc. Lansdale, PA, USA. 